on the eighth day of the cycle the perineum undergoes deturgescences A large cyst is produced in one ovary and massive atresia of follicles in both.

The second or third cycle succeeding those which were disturbed experimentally may not deviate very much from the normal.

Estradiol benzoate, progesterone or a combination of both hormones given to normal adult baboons in the first part of the cycle causes widespread ovarian damage, albeit, perhaps, of a temporary nature.

Department of Anatomy, University of the Witwatersrand, Johannesburg. March 21.

'Gillman, Joseph, Endocrinology, 26, 80, 434 (1940).

\section{Time as an Imaginary Fourth Dimension of Space is Incoherent}

Ir may be profitable in relation to current paradox to inquire very briefly into the relation of time to space. Consider two adjacent localities, one around a centre $P$ and the other around a centre $Q$. The familiar conical diagram asserts that for any sublocality that is within a certain cone centring on $P$ the relative time is real, while outside that cone it is pure imaginary; and likewise for a cone centring on $Q$. These cones intersect : and there are regions $R$, inside one of them and outside the other, for which time is real in relation to $P$ and imaginary in relation to $Q$, or, in current language, the region around $R$ is time-like in relation to $P$ but space-like in relation to $Q$. This is something far more complex than the properties of a simple continuum such as a practicable space.

The paradox here suggested may be traced back into the ultimate foundations. The doctrine of the Principle of Special Relativity has been condensed into the slogan that "Henceforth Time and Space are no more". The mathematical Theory of General Relativity has been built up around the postulate (memoir of 1916, English edition, § 3, p. 117) that "the general laws of nature are to be expressed by equations which hold good for all systems of coordinates, that is, are covariant with respect to any substitutions whatever (generally covariant)".

The present critique has been stimulated by the observational result that the vast universe of the spiral nebulæ may be in a state of uniform expansion", for this suggests the question to determine the formulø which express that expansion, as the formulæ of the Lorentz transformation express a uniform motion of translation. To my surprise, there appears to be no such set of formulæ. Thus permanent uniform expansion is impossible, so that in the course of time this expanding universe will cease to be uniform; which, by the way, is an alternative mode of accounting for the actual defect from uniformity that has been recently announced. But much more than this, the restriction of permanent uniformity to uniform translatory motion of the frame of reference carries us 'back to Newton', as the phrase goes, and may be even held to provide the explanation of this restricted indeterminacy of his frame of reference in space and time.

All such restrictions appear to be in flat contradiction to the postulate quoted above of General Relativity. The explanation is perhaps not far to seek : for if there is no longer time or space, there cannot any longer be velocity as the mode of expressing motion. But the formulæ here sought, and relations expressed in terms of them, would involve the velocity of one frame of reference relative to the other. Therefore such relations could not express laws of Nature : and these laws would have to be reformulated in terms of variables which probably have no intuitive relation to knowledge based on observation as given by our actual senses. The purely mathematical constructs of Ricci, now named tensors, are perhaps intended to be the foundation of a (very limited) formal universe expressed by variable of this kind. Meantime, the present astronomy, the queen of the mathematical sciences, would remain provisionally.

The reply to this critique might be to ask what is the alternative. For the tensor theory has led to a fow results, of extremely special type, some of which have been in part confirmed. An answer appears to be that the tensor theory is far too wide a frame for the actual universe of Nature, which involves six freedoms, three for position and three for motion, whereas the tensor framework works with ten variables (potentials), so that the universe of Nature would have room, so to say, to rattle about in it, while as regards some of the specially simple test questions that are practicable the four extra variables may not come into play.

There can be a further claim, that a systematic doctrine of a duly restricted progress of dynamical Action of the system in time, can profitably absorb the valuable elements of these theories which are a natural general foundation for physies, and of the wave-mechanical as well, by going back a century to utilize W. R. Hamilton's procedures.

Holywood,

JOSEPH LARMOR.

Northern Ireland. April 7.

2 NATURE, 147, 175 (1941).

\section{Malaria in War}

WITH reference to the interesting paper on malaria by Dr. V. B. Wigglesworth in NATURE of April 12, p. 436, I offer a short account of the methods used at Taranto during the War of 1914-18.

In 1917 the lines of communication for Salonika, etc., were taken through France and Italy to Taranto. In that year the rest camp at Taranto was hurriedly organized and with some 60,000 men passing through there were some 600 cases of malaria. In the winter of 1917-18 it was decided to deal with mosquitoes there; and to deal with all swamps, etc., within 2,000 yards of the camp. Colonel Robertson, R.A.M.C., was our expert, with great experience in India, of such work.

As Director of Works, Italy, I wired to the Director of Works, France, "Send me your best Land Drainage expert for Taranto." He replied, "You have got him-try Kenworthy" (who was then in charge of R.E. Stores at the base). Major Kenworthy in civil life was a land drainage consulting engineer.

Kenworthy with his experienced eye at once saw that excellent work had been done by the Italians; but that owing to a technical omission the ditches had been allowed to relapse. So he cleaned them of growths; and dug a smaller trench at the bottom of small section to deal with low-water flow and 